\title{
Note on an Abstract Inversion Principle
}

\author{
By L. C. Hsu
}

(Received 8th November, 1950. Read 1st December. 1950)

In a recent paper [1] of Bell, an abstract inversion principle has been formulated for inverting a type of finite series by employing operators. Bell's result involves Baker's general principle of cross-classification [2], Dedekind-Möbius inversion, L.C.M. inversion and some known generalisations. The purpose of this note is to introduce operators of negative degree and to formulate an inversion principle which covers more cases than Bell's.

1. With Bell's notation, $X_{t}$ denotes a subset of precisely $t$ distinct elements taken from an abstract set $\left\{x_{1}, x_{2}, \ldots, x_{n}\right\}$, and $X_{n-t}^{\prime}$ is the complementary subset; $f_{i}\left(X_{l}, X_{n-t}^{\prime}\right), F_{t}\left(X_{t}, X_{n-t}^{\prime}\right)$ denote arbitrary singlevalued functions of $\left(X_{t}, X_{n-1}^{\prime}\right)$ whose values belong to a module $M . P(T)$ denotes an arbitrary polynomial in operators $T$, which are defined by

$$
T_{s} f_{t}\left(X_{l}, X_{n-t}^{\prime}\right)= \begin{cases}f_{t-1}\left(X_{t-1}, X_{n-t+1}^{\prime}\right) & \text { if } x_{s} \varepsilon X_{t} \\ 0 & \text { otherwise }\end{cases}
$$

where $X_{t-1}$ is the result of deleting $x_{s}$ from $X_{t}$, and $T_{0}$ is the identity operation. The product $T_{t} T_{s}$ means operating first with $T_{s}$ and then with $T$. Obviously, both the commutative law and the associative low hold for $T$ operators, and for $s>0$

$$
T, 2 f_{t}\left(X_{t}, X_{n-t}^{\prime}\right)=0(0 \text { in } M), \quad T_{a}^{2}=O \text { (zero operator). }
$$

2. Whenever $x_{\varepsilon} \varepsilon X_{\ell}$, Bell's operator $T_{s}$ is just an operation of transferring an element from $X_{t}$ into $X_{n-t}^{\prime}$. Thus it is also possible and quite natural to introduce operators of negative degree by the following reverse relation:

$$
T_{s}^{-1} f_{l}\left(X_{l}, X_{n-l}^{\prime}\right)= \begin{cases}f_{l+1}\left(X_{l+1}, X_{n-1-1}^{\prime}\right) & \text { if } x_{s} \varepsilon X_{n-l}^{\prime}, \\ 0 & \text { otherwise. }\end{cases}
$$

Here $T_{s}^{-1}$ means transferring the element $x_{s}$ from $X_{n-l}^{\prime}$ back to $X_{i}$ whenever $x_{\varepsilon} \varepsilon X_{n-i^{*}}^{\prime}$ In particular, $T_{0}^{-1}=T_{0}, T_{\star}^{-1} T_{\star}^{-1}=T_{s}^{-2}=O(s>0)$. 
Now evidently the commutative law does not generally hold, e.g. for $s>0, T_{s}^{-1} T_{8} \neq T_{s} T_{s}^{-1}$. But except in the case just mentioned, the commutative law always holds. The fact that the associative law generally holds is easily seen.

Let $P(T)$ and $Q(T)$ be any two polynomials in operators with coefficients in a commutative ring $C$. If $T_{s}$ and $T_{s}^{-1}$ (with coefficients in $C$ ) appear in $P(T)$ and $Q(T)$ respectively, then we say that there is a pair in $P, Q$, where $s$ is called the index of $T_{s}$ or $T_{s}^{-1}$. The following commutative law may sometimes be useful:

If there is no pair occurring in $P, Q$, then we always have

$$
P(T) Q(T)=Q(T) P(T) .
$$

3. Let $P(T)$ be an arbitrary polynomial in operators $T_{s}^{\prime}, T_{s}^{-1}$, etc., with coefficients in $C$. We shall say that $P^{-1}\left(T^{\prime}\right)$ is the inverse (polynomial) of $P(T)$ if and only if

$$
P(T) P^{-1}(T)=T_{0} .
$$

Simple examples are ${ }^{1}$ :

(i) $P(T)=T_{s}+T_{s}^{-1}, \quad P^{-1}(T)=T_{s}+T_{s}^{-1} \quad(s>0)$.

(ii) $P_{1}(T)=T_{0}+T_{1}+T_{2}^{-1}+T_{3}^{-1}$,

$$
P_{1}^{-1}(T)=T_{0}-T_{1}-T_{2}^{-1}-T_{3}^{-1}+2\left(T_{1} T_{2}^{-1}+T_{1} T_{3}^{-1}+T_{2}^{-1} T_{3}^{-1}\right)
$$

$P(T)$ may have no inverse, e.g.

$$
\left(T_{0}+T_{1}+T_{1}^{-1}\right)\left(T_{0}-T_{1}-T_{1}^{-1}\right)=T_{0}^{2}-\left(T_{1}+T_{1}^{-1}\right)^{2}=T_{0}-T_{0}=O
$$

so that both factors on the left-hand side are zero divisors having no inverses.

In particular, if $P(T)=T_{0}+Q(T)$, where $Q(T)$ does not contain $T_{0}$ and the indexes of all $T$ 's in $Q(T)$ are distinct, then the inverse polynomial $P^{-1}(T)$ always exists and may easily be determined by expanding $\left(T_{0}+Q(T)\right)^{-1}$ formally by the binomial theorem. In fact, in the present case both the associative law and the commutative law do hold.

4. Now Bell's abstract inversion principle can easily be extended to the following form :

Let $P(T)=P\left(T_{1}, T_{1}^{-1}, \ldots, T_{n}, T_{n}^{-1}\right)$ be an arbitrary polynomial operator such that its inverse $P^{-1}(T)=\left(P\left(T_{1}, T_{1}^{-1}, \ldots, T_{n}, T_{n}^{-1}\right)\right)^{-1}$ exists. Then

1 Example (i) follows immediately from the fact that $T_{4}^{-1} T_{\triangleleft}+T_{\star} T_{a}^{-1}=T_{0}(s>0)$. 
the following two sets of equations are equivalent:

$$
\begin{gathered}
F_{l}\left(X_{t}, X_{n-\ell}^{\prime}\right)=P(T) f_{t}\left(X_{t}, X_{n-t}^{\prime}\right) \quad(t=0,1, \ldots, n), \\
f_{l}\left(X_{\ell}, X_{n-l}^{\prime}\right)=P^{-1}(T) F_{\ell}\left(X_{t}, X_{n-t}^{\prime}\right) \quad(t=0,1, \ldots, n) .
\end{gathered}
$$

To see that the extension is genuine, it suffices to observe the simple inversion

$$
\begin{aligned}
& F_{t}\left(X_{t}, X_{n-\ell}^{\prime}\right)=\left\{\prod_{s=1}^{n}\left(T_{s}+T_{s}^{-1}\right)\right\} f_{l}\left(X_{l}, X_{n-t}^{\prime}\right) ; \\
& f_{t}\left(X_{t}, X_{n-\ell}^{\prime}\right)=\left\{\prod_{s=1}^{n}\left(T_{s}+T_{s}^{-1}\right)\right\} F_{l}\left(X_{t}, X_{n-t}^{\prime}\right),
\end{aligned}
$$

which is obviously not obtainable from the original inversion principle of Baker and Bell.

Note that in our case the commutative law has been sacrificed, so that the relation $P^{-1}(T) P(T)=P(T) P^{-1}(T)=T_{0}$ requires justification. Evidently (4.1) is obtainable by operating with $P(T)$ on both sides of (4.2) and using the definition of $P^{-1}(T)$. Since (4.2) is a system of $2^{n}$ simultaneous equations having the solution (inverse) (4.1) it is clear that the corresponding matrices of (4.2) and of (4.1) are non-singular. Thus it follows that (4.2) must be also the unique solution of (4.1). This shows that (4.1) and (4.2) are equivalent, and consequently

$$
P^{-1} P=P P^{-1}=T_{0} \text {. }
$$

5. The inversion principle may easily be further extended by considering $k$ subsets of $X_{n}(k \geqslant 2)$ and defining operators of transference between each pair of subsets. Finally, it may be worthy of mention that P. Hall's enumeration principle [3] or its special case Weisner's inversion formulae [4] cannot be deduced from the abstract inversion principle here discussed. On the other hand, Hall's inversion formula also does not include the type of inversion considered in this note. Detailed explanations will be omitted here.

\section{REFERENCES.}

[1] Bell, E. T., Duke Math. Journal, 15 (1948), 79-85.

[2] Baker, H. F., Proc. London Math. Soc., 21 (1891), 30-32.

[3] Hall, P., Quarterly Journal of Math. (Oxford), 7 (1936), 134-151.

[4] Weisner, L., Trans. American Math. Soc., 38 (1935), 485-492, 474-484.

Magdalene College, CAMBRIDGe, 